\title{
Improved Genetic Algorithm to Extract the Edge of the Selected Threshold
}

\author{
Zhao Xiaofeng \\ Shandong Women's University \\ zhxf0929@sina.com
}

\begin{abstract}
Threshold is presented according to the principle of genetic algorithm, the secondary search optimization of the improved genetic algorithm is divided into two times to seek the global optimal solution, namely using the results of the search for solution for the first time to determine the second optimization selection of initial population, as a result of the optimization may be given for the first time is not necessarily the global optimal solution, but it certainly is a good result, the optimization process will be the second time, the initial population of restrictions in a neighborhood of the search results for the first time. The simulation results proved the advantage of the proposed algorithm.
\end{abstract}

Keywords: genetic algorithm; selected threshold; edge detection; image denoising

\section{Introduction}

With the rapid development of computer technology and multimedia technology, digital image at the beginning of the human life and activities play an increasingly important role, such as satellite TV, X-ray, astronomical observation, the development of geographic information systems are used in such fields as digital image, image processing technology has been rapid development, therefore, the image segmentation is the basis of image processing technology [1]. Edge detection and denoising is an important technology in image segmentation, image denoising processing can improve the image quality, image edge profile is carrying a lot of information, therefore, image denoising and edge detection for the accurate analysis of image information, achieve precise segmentation provides an important guarantee of [2]. In acquiring stage often introduce a lot of noise, so before the image further processing, usually need to the image noise reduction processing. Image noise reduction has become a classic in the field of image processing problems, several ten years of research history, however, the traditional denoising method is accomplished very hard can accurately locate edges, the edge of the reaction more details, and also can filter out the noise as much as possible. In edge detection, and if we can successfully extract the image edge, then image analysis will be simplified, image recognition would be much easier, but a lot of images and no concrete objects, understanding of the images depends on their texture properties, edge detection and extraction of the texture and properties and has a very close relationship. Therefore, the research on edge detection algorithm also has the extremely vital significance.

Sources of noise in digital image there are two major categories: electronic noise and light electronic noise. Electronic noise is impedance in the electronic device caused by electronic random thermal motion. This kind of noise can be zero mean gaussian white noise as its model, it has a gaussian function in the shape of

1. The Project of Natural Science Foundation,Shandong Province. ZR2012FL04. Design and Arithmetic Research of Image Sparse Dictionary.

2. The Project of Natural Science Foundation,Shandong Province. ZR2013FL024. Contour Grouping Method Based On Particle Filter and Its Application in Image Retrieval. 
histogram distribution and flat power spectrum, can be used to fully express its variance. Photoelectron noise is caused by the photoelectric conversion process statistical properties of the light. In the case of weak light, this kind of noise is more serious, the commonly used with poisson distribution of random variables as photoelectron noise model. When light is strong, this kind of noise tends to gaussian distribution [3]. Generally, the image denoising technology is divided into two categories: [2] denoising in the spatial domain and transform domain denoising. Refers to the image plane itself in the spatial domain, this kind of method of image pixels directly for processing. Transform domain denoising method refers to the image transformation, in the transform domain of image processing, transform domain coefficients of inverse transformation of the reentry after processed, ultimately after denoising image. Currently the most used transform method is Fourier transform and wavelet transform. The simplest space domain image denoising method is average filter. Mean filter in filtering the noise at the same time, also to remove the image details, discontinuous step transform in the image smooth into a slow change, make the image fuzzy. To minimize the total variation method is also a high-profile spatial domain filtering method, but this method is easy to smooth out the detail of the image information. Image denoising based on partial differential equation is also - class spatial domain filtering method, this method has very strong inhibition to the impulse noise, but the white gaussian noise denoising effect. Every kind of mathematical tools appear to attract people to try and use it to solve the problem of various interest, it is also the introduction of these algorithms in image denoising technology has made great progress, and also for the further study on this field has provided a broad space for development.

Image is an important source of human and exchange information, therefore, the application of image edge processing inevitably involves all aspects of human life and work. With the expansion of the scope of human activities, the image edge detection and extraction processing of the application field also has been widely used. Digital Image edge detection (Digital Image Processing) refers to converting Image signal into Digital signal and use the computer to deal with the process.

\section{Related Works}

Signal to change over time, often using two of the most basic way of description, the time domain and frequency domain. Time domain to describe the change of signal strength over time and frequency domain is described in a certain time range of the signal frequency distribution. Image denoising technology basically can be divided into noise in spatial domain and transform domain noise control two kinds big. Refers to the image plane itself in the spatial domain, this kind of method is to directly in the original image for data operation, to deal with gray levels of pixels. Transform domain refers to the image transformation noise reduction method, in the transform domain image transform domain coefficient to carry on the corresponding processing, inverse transformation of the reentry after processed, after get noise image.

Median filter is a nonlinear filtering, is based on the theory of order statistics of a nonlinear signal processing technology can effectively restrain noise. Because it in the whole computing process does not need image statistical features, it is more convenient to use so. Median filtering the first application in one-dimensional signal processing technology, then applied to a two-dimensional image signal processing. This method can overcome the neighborhood smoothing filter etc caused by the linear filter in the process of smoothing image detail, and to filter out pulse interference and noise image scanning is the most effective. But for some image detail more, especially dot, line, spire details more images are not suitable for using 
median filter method. The basic principle of median filter is a pixel neighboring domain of each pixel values sorted according to the gray value, and then select the sequence of intermediate value instead of the original grey value of pixels, so as to eliminate the role of the isolated noise points.

Wavelet analysis the core idea is: according to the scale to analyze the signal to the wavelet scaling and translation, and then to grind Gui signal and the correlation between the wavelet. It's like to observe an object from different distance, signal after stretching (called the large scale) of wavelet correlation reveals is rough features of signals. Signal after the contraction (known as the small scale) is a signal of wavelet correlation reveals fine features. So it is both signal wavelet analysis can say see trees and forests. Multi-resolution analysis is the essence of the wavelet analysis. Using wavelet transform processing in engineering application, is the most widely used binary wavelet transform, the scale parameter discretization in it, for the translation on the time domain parameter keep continuous transformation, which does not destroy the signal in time domain on the translation parameters. At present, the wavelet image denoising method has become an important branch and the main research Gui denoising direction, in the past ten years, the wavelet method in signal and image denoising applications of scholars widely attention, including the method of using wavelet transform for image noise reduction of the most commonly used method for threshold denoising method. The value is the basic principle of denoising, image signal after wavelet decomposition, the smaller the coefficient is generally noise, while larger wavelet coefficients for the most part is given priority to with practical signals. So can set the appropriate part of high frequency coefficient threshold, the new wavelet coefficients obtained after thresholding function mapping, again to the new wavelet coefficients wavelet reconstruction. In the threshold denoising method, two is the most basic key element of the threshold and the threshold value function.

Edge detection is all based on boundary integral method is the most basic processing. Edges of the image focused image, most of the information is the important foundation of image segmentation depends on, is the important basis texture analysis and image recognition. The determination of image edge and extraction for the entire image scene recognition and understanding is very important.

First introduce some basic steps of image edge detection [5]:

(1) filter. Edge detection is mainly based on the derivative calculation, but the calculation of derivative is sensitive to noise. Filter to reduce the noise at the same time also makes the image edge intensity is reduced.

(2).Enhancement algorithm is to adjacent domain or local grey value has highlighted some significant changes. General gradient amplitude by calculation.

(3) detection. There are many points in the image of gradient amplitude is large, and these points are not necessarily is edge, how to determine the real edge points, the most simple edge detection method is determined by gradient amplitude threshold.

(4) positioning. On the edge of the location estimation in the sub pixel resolution, on the edge of the bearing can also be estimated.

\section{System Model Description}

In any space function $f(t)$, namely $\forall f(t) \in M^{2}(R)$, the continuous wavelet transform (sometimes referred to as the integral wavelet transform) is defined as

$$
W T_{f}(a, b)=|a|^{-1 / 2} \int_{-\infty}^{\infty} f(t) \bar{\psi}\left(\frac{t-b}{a}\right) d t
$$


where

$$
\Psi_{a, b}(t)=|a|^{-1 / 2} \psi\left(\frac{t-b}{a}\right)
$$

Any transformation must exist inverse transformation only has the practical significance, but not all of the inverse transformation transform. For wavelet, wavelet must satisfy (2) adopted by the type of permit conditions, inverse transform didn't exist.

In practical applications, especially in the computer realization of wavelet transform, the continuous wavelet transform must be discretization. So, we usually put the continuous wavelet transform discretization of mesoscale factor a and translation factor were taken as follows: $\mathrm{a}=\mathrm{a}_{\mathrm{j}}, \mathrm{b}=b=k a_{0}^{J} b_{0}$, among them, the step size is a fixed value. In order to facilitate research, generally assumed $a_{0}>1,, b_{0}>1$. Then the corresponding discrete wavelet can be defined as

$$
\Psi_{j, k}(t)=\left|a_{0}\right|^{-1 / 2} \bar{\psi}\left(a_{0}^{-j} t-k b_{0}\right)
$$

In Gui research problems in digital image processing theory of multi-scale analysis of wavelet transform, the establishment of this theory for the construction of wavelet base provides a simple method, and thus puts forward the current widespread use of Mallat fast wavelet decomposition and reconstruction algorithm, this kind of thought and sampling filter groups more idea coincides with mine, so the multi-scale analysis in the wavelet transform theory has very important position. In image pyramid decomposition algorithm (Pyramidal algorithm), inspired by combining multi-scale analysis, put forward the signal tower multi-scale decomposition and synthesis algorithms, discrete wavelet using mallat algorithm rapid implementation of the general, it will be the combination of wavelet transform and multi-scale analysis of organic. Wavelet multi-scale edge detection algorithm is to use a smooth function, at different scales to smooth processing to detect the signal, and then based on the wavelet transform of signal after the smooth coefficient of the first or second order derivative to find the abrupt change point of the signal. Meet with two-dimensional smoothing function $\theta(x, y)$

$$
\begin{array}{r}
\iint \theta(x, y) d x d y=1 \\
\theta_{s}(x, y)=\frac{1}{s} \theta\left(\frac{x}{s}, \frac{y}{s}\right)
\end{array}
$$

If $f_{j}=V_{j}^{2}$ the said image function $\mathrm{f}$ at scale $2^{\mathrm{j}}$ looming, namely $\mathrm{f}$ rough like, said the approximation error, namely/error, according to $2 \mathrm{~d}$ image multi-scale analysis and Mallat algorithm, $d_{j} \in W_{j}^{2}$ can get the image wavelet decomposition

$$
f_{0}=f_{1}+d_{1}=f_{2}+d_{2}+d_{1}=f_{3}+d_{3}+d_{2}+d_{1}
$$

Because it is $2 \mathrm{~d}$ image multi-scale $d_{j}$ analysis, therefore, on behalf of the three sub image details of image wavelet decomposition method to construct the image edge details of the main idea is:

Leave the low-frequency raw sugar $f_{n}$ like $f$, then use high frequency detail $d_{n} \cdots d_{1}$ of two-dimensional image reconstruction, and then to edge extraction of the fused images. Because images/after wavelet decomposition, after smooth filtering, the low frequency part, after high frequency filter, the high frequency part, in wavelet decomposition approximation coefficients section, contains the detail of the image information is more and more small, image edge information is decompose step by step to the high frequency part. So can be resolved through the information of high frequency part of the refactoring to extract image edge. 


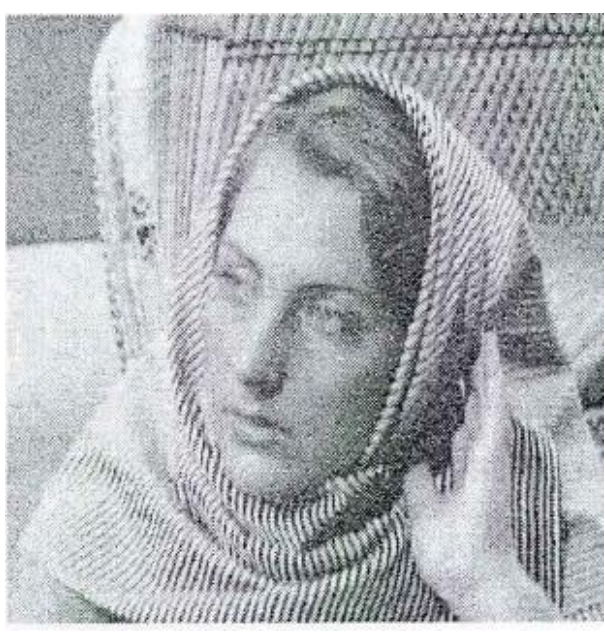

(a) The Original Image

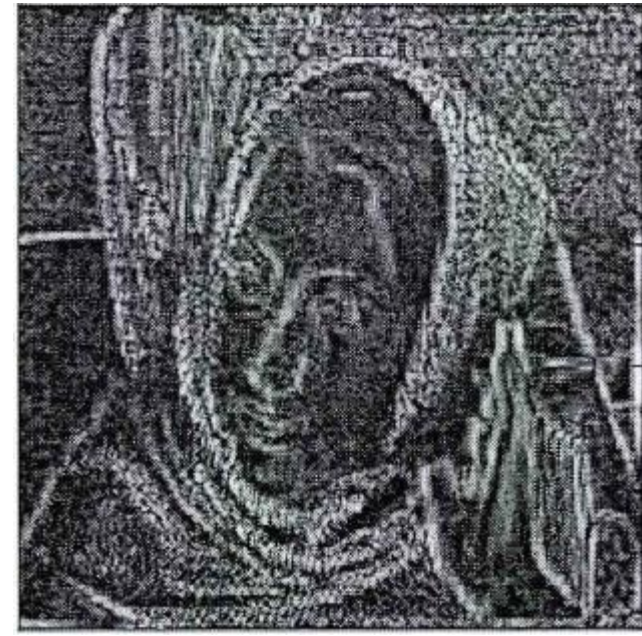

(b) Reconsitution

Figure 1. Image Reconstruction

Species survival process generally follows the rule of the evolution of Darwin's natural selection, survival of the fittest. Individual in the population's ability to adapt to the environment of different selection or eliminated by nature. Evolution results reflected in the individual structure of the chromosome contains a number of genes, and the corresponding phenotype and genotype of contact embodies the external characteristic of the individual and the logical relationship between the internal mechanism. Through selection, crossover and mutation between individuals living to adapt to nature environment. Biological chromosomes represented in mathematical way is a string of digital, also known as chromosomes; Can be measured by chromosomal numerical adaptable. The choice of the chromosome or eliminate problems can also be according to the maximum or minimum problem.

Genetic algorithm simulates the genetic and evolutionary process of organisms in the natural environment, and form a kind of adaptive global optimization probability search algorithm, which is a kind of mathematical simulation of biological evolution process, is also one of the most important form of evolutionary computation. Genetic algorithm in which the solution of the problem can be expressed as "chromosome", on the basis of the principle of survival of the fittest, pick out to adapt to the environment of "chromosome" replicate, again through crossover and mutation two operations to produce a new generation of "chromosome" is more suitable for the environment, so that generations of improvement, the result was one of the most suitable for environment, also get the optimal solution of problem.

Genetic algorithm is compared with other optimization algorithms, the advantage is that it depends on the solution of solving the target problem completely individual and fitness of the space, without other additional conditions, so it is for solving complicated nonlinear and non-structural has a strong ability to solve problems, but the use of traditional genetic algorithm to select the optimal edge detection threshold, there are defects such as slow convergence speed and early into the convergence and optimization results and the optimal solution is large. So this section of the traditional genetic algorithm was improved, the improved genetic algorithm not only improve the convergence speed of the algorithm and the optimization result is very close to the optimal solution. This main idea is that the improved genetic algorithm is divided into two times to seek the global optimal solution, namely using the results of the search for solution for the first time to determine the second optimization selection of initial population, as a result of the optimization may be given for the first time is not necessarily the global optimal 
solution, but it certainly is a good result, so we can limit the second optimization process of the initial population for the first time in a neighborhood search results. At this point the second optimization of initial population fitness is higher. According to the genetic theory, two genes are excellent individuals, the possibility of their offspring is excellent than general of the offspring of two individuals to produce, so in theory, this algorithm is feasible, and more conducive to search to the global optimal solution. Here we selected the between-cluster variance calculation function as the fitness function, the calculation formula for the concrete

$$
\sigma^{2}(t)=w_{1}(t) w_{1}(t)\left(u_{1}(t)-u_{2}(t)\right)^{2}
$$

Where $\mathrm{t}$ means to deal with image threshold, $w_{1}(t)$ said the image grey value is less than the threshold value of $\mathrm{f}$ number; $w_{2}(t)$ Said the image grey value is greater than the threshold $t$ sizes; $u_{1}(t)$ Said grey value is less than the threshold $t$ all pixels in the image of the average gray level values; Said the image grey value is greater than the threshold $t$ all pixels of the average gray level values; Through the above mentioned two optimization genetic algorithm, to find out $t$ value to be biggest, is the best threshold $\mathrm{T}$, there are

$$
T=\arg \max \sigma^{2}(t)
$$

\section{Simulation Results and Analysis}

First of all, we respectively to the standard image woman, lenna, Belmont images to calculate. First of all, using wavelet decomposition of the original images are decomposed, then use type (8) in high frequency image reconstruction, get fringe figure, finally USES the above in this paper, the improved genetic algorithm for automatic selection of threshold value, and then with a selection of threshold value of image broadly value processing, so as to get the edge image.

Algorithm in the detection of woman image, this paper compared canny algorithm has better ability to suppress noise, and compared with the modulus maxima multiscale edge detection algorithm, can filter the noise in ability is outstanding, but improved algorithm in detail and filter the false edge edge detection ability is more apparent, the detected image better.

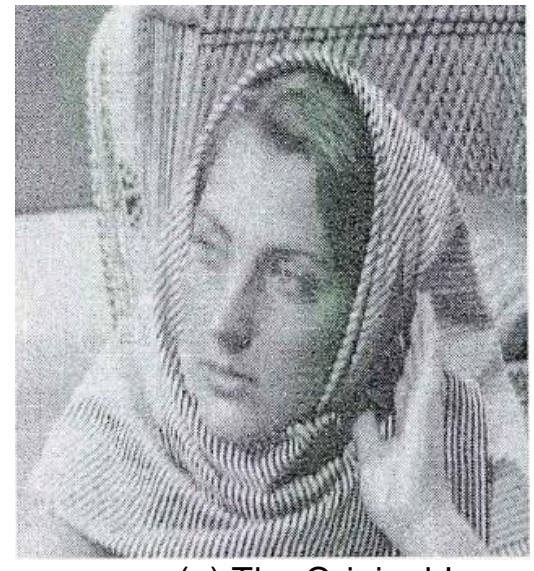

(a) The Original Image

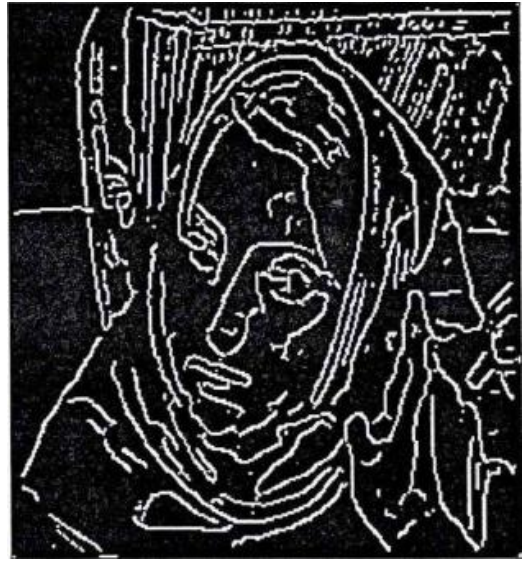

(b) Wavelet 

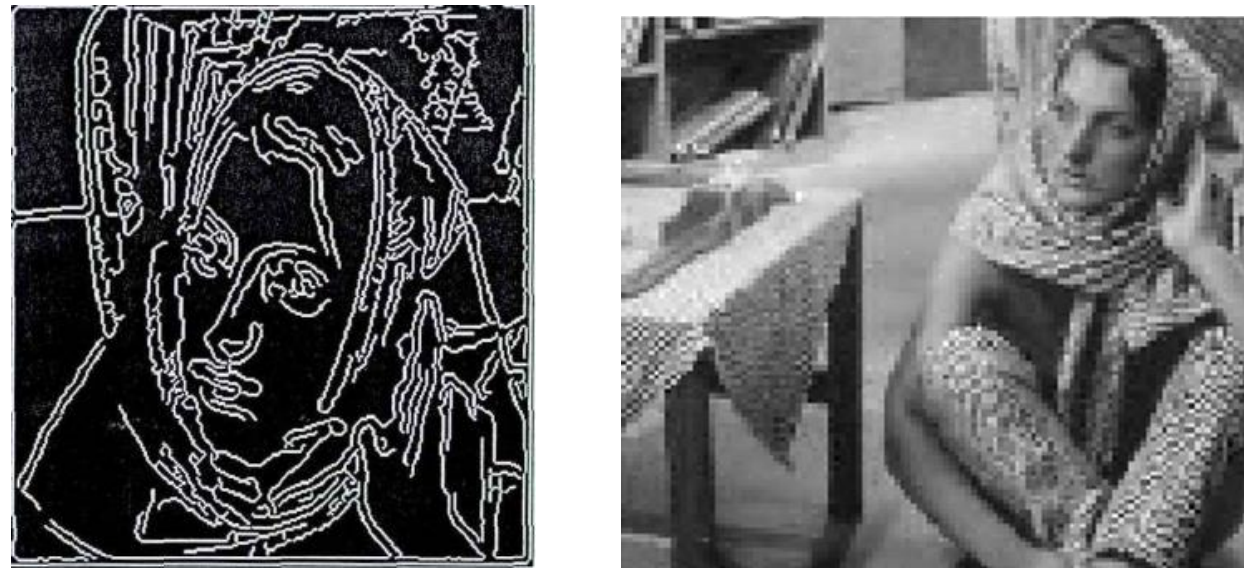

(c) Multi-scale Edge Detection Image

(d) Proposed

Figure 2. Various Algorithms Compared of Woman

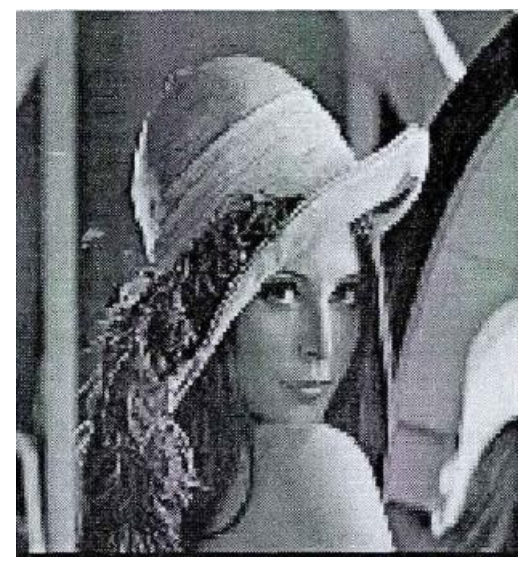

(a) The Original Image

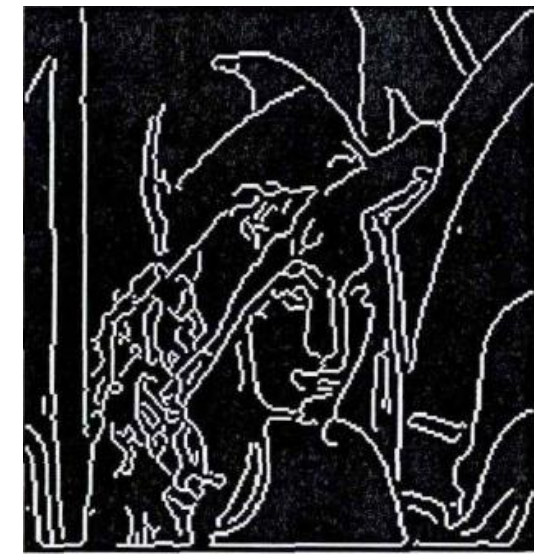

(c) Multi-scale Edge Detection Image

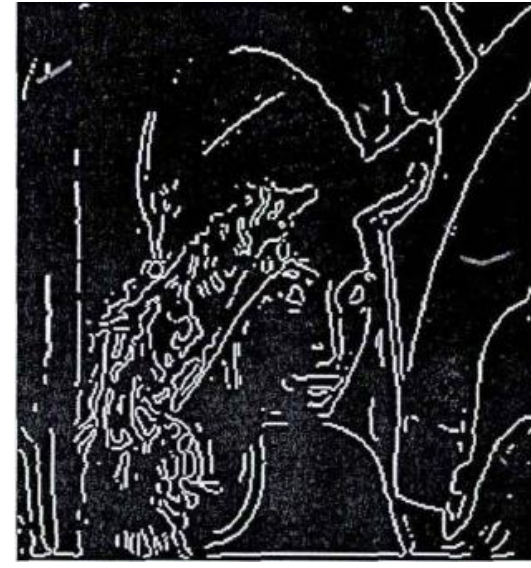

(b) Wavelet

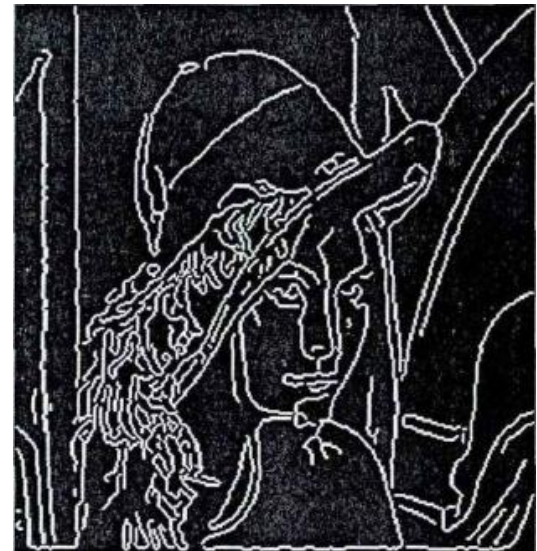

(d)Proposed

Figure 3. Various Algorithms Compared of Lenna

\section{Conclusion}

This paper introduces the more cutting-edge technology of image edge detection based on wavelet transform modulus maxima multi-scale edge detection algorithm. Based on this algorithm, this paper puts forward the multi-level wavelet decomposition details edge detection method and the improved genetic algorithm with the combination of the new 
algorithm, and through simulation comparison, can see clearly that the new algorithm of edge detection and significantly than canny algorithm based on wavelet transform modulus maxima multi-scale edge detection algorithm is good, can not only well extract the edge of the complete information, and the edges of the extract has the very good continuity, but also can filter the noise and remove the false edge, at the same time shows the proposed methods in image edge detection has higher positioning accuracy and better denoising ability, also prove the validity of the algorithm is given, also for the future to the selection of image edge detection algorithm provides guiding significance.

\section{References}

[1] A. F. Abdelnour and I. W. Selesnick, "Nearly symmetric orthogonal wavelet bases", Proc. IEEE Int. Conf. Acoust., peech, Signal Processing (ICASSP), (2001).

[2] G. Beylkin, "On the representation of operators in bases ofcompactly supported wavelets SlAM", J. Numer.Anal, vol. 29, no. 6, (1992), pp. 1716-1740.

[3] J. A. Bilmes, "A gentle tutorial of the EM algorithm and its application to parameter estimation for gaussian mixture and hidden markov models", International Cmputer Science Institute, (1998).

[4] T. Bulow, "Hypercomplex spectral signal representations for the processing and analysis of images", $\mathrm{Ph}$. D. dissertation, Christian AlbrechtsUniv, (1999); Kiel, Germany.

[5] M.Bahri, "Construction quarternion-valued wavelets", Mathematica, vol. 26, no. 1, (2010), pp. 107114.

[6] B. Bayraktar and M. Analoui, "Image denoising via fundamental anisotropic diffusion and wavelet shrinkage: a comparative study computational imaging II”, Proceeding of SPIE-IS \& T Electric Imaging, vol. 5299, (2004), pp.387-398.

[7] M. Bahri, R. Ashino and R. Vaillancourt, "Two-dimensionalquaternion wavelet transform Applied Mathematics and Computation", vol. 218, (2011), pp. 10-21.

[8] T. D. Bui and G. Y. Chen, "Translation-invariant denoising using multiwavelets", IEEE Trans. Signal Processing, vol. 46, no. 12, (1998), pp. 3414-3420.

[9] A. G. Bruce and H. Y. Gao, "Understanding wave Shrink: variance and bias estimation, Biometrika, vol. 83, no. 4, (1996), pp. 727-745.

[10] W. Bender, D. Gruhl and N. Morimoto, "Techniques for Data Hidding”, Proceedings of the SPIE 2420", storage and retrieval for image and video database, no. 3, (1995), pp. 164-173.

\section{Author}

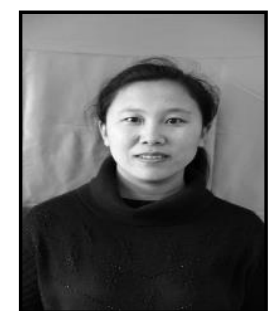

Zhao Xiaofeng obtained Bachelor's Degree of Science from Shandong Normal University in 2004, and obtained Master's Degree of Engineering from Shandong University in 2011. She is currently researching on Digital Image Processing Theory and Application. 